\title{
An Integrated Strategy for the Cultural Adaptation of Evidence-Based Interventions
}

\author{
Souraya Sidani ${ }^{*}$, Sarah Ibrahim², Jana Lok ${ }^{3}$, Lifeng Fan4, Mary Fox ${ }^{5}$, Sepali Guruge ${ }^{6}$ \\ ${ }^{1}$ Canada Research Chair School of Nursing Ryerson University, Toronto, Canada \\ ${ }^{2}$ Arthur Labatt Family School of Nursing University of Western Ontario, London, Canada \\ ${ }^{3}$ Lawrence S. Bloomberg Faculty of Nursing University of Toronto, Toronto, Canada \\ ${ }^{4}$ Toronto Chronic Diseases Centre, Toronto, Canada \\ ${ }^{5}$ School of Nursing York University, Toronto, Canada \\ ${ }^{6}$ School of Nursing Ryerson University, Toronto, Canada \\ Email: ^ssidani@ryerson.ca, sibrah25@uwo.ca, jana.gegus@utoronto.ca, lifeng.fan@alum.utoronto.ca,maryfox@yorku.ca, \\ sguruge@ryerson.ca
}

How to cite this paper: Sidani, S., Ibrahim, S., Lok, J., Fan, L.F., Fox, M. and Guruge, S. (2017) An Integrated Strategy for the Cultural Adaptation of Evidence-Based Interventions. Health, 9, 738-755.

https://doi.org/10.4236/health.2017.94053

Received: March 3, 2017

Accepted: April 27, 2017

Published: April 30, 2017

Copyright (C) 2017 by authors and Scientific Research Publishing Inc. This work is licensed under the Creative Commons Attribution International License (CC BY 4.0).

http://creativecommons.org/licenses/by/4.0/

\begin{abstract}
Background: The importance of adapting evidence-based health interventions to enhance their congruence with the beliefs of ethno-cultural communities is well recognized. Although a systematic cultural adaptation process is available, it lacks specific instructions on how to adapt interventions so that they are aligned with cultural beliefs. In this paper, we present an integrated strategy that operationalizes the adaptation process by describing specific practical instructions on how to align interventions with cultural beliefs. Methods: The strategy integrates concept and intervention mapping, and uses mixed methods for gathering data from community representatives. The data pertain to a community's cultural beliefs and values related to a health problem, acceptability of evidence-based interventions targeting the problem, and aspects of the interventions that should be modified to enhance their fit with cultural beliefs. A step-by-step protocol is described to guide application of the integrated strategy for cultural adaptation. Conclusions: The strength of the integrated strategy relies on the use of concept and intervention mapping approaches for specifying a stepby-step protocol to actively engage community representatives in the cultural adaptation of interventions. Future research should evaluate the utility of this strategy.
\end{abstract}

\section{Keywords}

Acceptability, Cultural Adaptation, Evidence-Based Practice, Health Interventions, Protocol 


\section{Introduction}

Evidence-based interventions (EBIs) are disseminated for use in daily practice, but their uptake may be limited if they are perceived as unacceptable [1]. Perceived acceptability is affected by beliefs about health problems and treatments that are shaped by cultural worldviews [2], which represent cognitive schemas that explain health or illness processes. These schemas influence one's view of health, experience and interpretation of health problems (e.g. causes and consequences of a problem), engagement in health behaviors (e.g. food selection and preparation), and treatment of health problems [3]. For instance, Givens, Houston, van Voorhees, Ford \& Cooper [4] reported that African, Asian and Hispanic Americans do not believe in biological causes of depression and hence, do not consider antidepressant medications as acceptable treatments.

When EBIs are unacceptable to an ethno-cultural community, there is often a discrepancy between the community's beliefs related to health problems targeted by the interventions, and those underlying the interventions [5]. Individuals within the community will not seek, initiate or adhere to these interventions, which hinder improvement in health outcomes and result in health disparities [6] [7]. Cultural adaptation of EBIs is proposed as a means to address these disparities. Cultural adaptation refers to the process of modifying EBIs to new ethno-cultural communities. New communities can include those for whom the interventions were not originally designed and for whom the effectiveness of the interventions was not evaluated. Modification is done to align the interventions with the culturally linked aspects of the community members' beliefs and experiences.

Many frameworks [8] [9] [10] [11] [12] [13] propose a planned, organized, and collaborative process for actively engaging representatives of the target ethnocultural community in the cultural adaptation of EBIs and suggest aspects of the culture to consider. As commonly described, the cultural adaptation process involves modifying non-specific elements of EBIs but not the specific elements. The non-specific elements refer to the format and activities that facilitate the implementation of the specific elements. The specific elements are the active ingredients responsible for triggering the mechanisms underlying the interventions' effects [14]. The goal is to align the interventions with a community's cultural beliefs, while maintaining the interventions' core components that operationalize their specific elements [3].

The frameworks suggest that adaptation consists of making changes in the interventions that are consistent with the deep and surface structures of the culture. The changes include: omitting, adding, or modifying some aspects of the non-specific elements of the interventions; these elements often include words and examples to relay the interventions' content, activities comprising them, mode or format for their implementation, and dose (e.g. number of sessions) at which they are delivered [12]. Deep structure reflects the beliefs, values, norms or worldviews held by an ethno-cultural community. The surface structure represents the superficial, observable aspects of culture such as language, food, 
metaphors, colors, and images [2] [3] [6].

The cultural adaptation process consists of three main steps: 1) assessing the community's cultural beliefs; 2) examining the fit between the community's beliefs and those underlying the EBI; and 3) determining the modifications to make in the intervention protocol and manual. The adapted intervention is then pilot-tested [6] [11] [12].

Although the literature identifies the steps of the cultural adaptation process, it falls short of providing specific instructions on how to implement them, leaving much room for variability in their interpretation, organization and implementation. In this paper, we present an innovative strategy that addresses this gap in practical knowledge guiding the implementation of the cultural adaptation process. The strategy integrates elements of concept and intervention mapping and provides specific, practical instructions for carrying out the three steps of the cultural adaptation process, illustrated with examples related to the health problem of low engagement (i.e. below the recommended level of 150 minutes per week) in physical activity.

\section{Integrated Strategy for Cultural Adaptation of Interventions}

The strategy for cultural adaptation of EBIs extends the three main steps of the process mentioned previously, by integrating elements of concept mapping and intervention mapping [15] and the approach for assessing the acceptability of interventions [16]. The strategy is consistent with the emphasis on involving community members in the adaptation process [15]. Concept mapping is a structured process that guides researchers in engaging community representatives to clarify their views of the health problem targeted by the EBIs, as experienced by members of the target ethno-cultural community [17]. Intervention mapping is a process for selecting evidence-based interventions that are feasible and acceptable to the community in addressing the health problem [15] [18]. Assessment of acceptability consists of appraising EBIs for their appropriateness in managing the health problem [19].

Some preparation is required prior to implementing the integrated strategy. This includes a review of pertinent literature to identify all possible indicators and determinants of the health problem as well as EBIs that address the health problem, its indicators and/or determinants.

The integrated strategy is implemented in three sequential steps (Figure 1). In step 1, representatives of the target ethno-cultural community are engaged in identifying the indicators and determinants of the health problem most relevant to, or consistent with, their cultural beliefs, as is usually done in concept mapping. Indicators and determinants identified as culturally relevant and for which EBIs are available are further discussed in step 2, as is done in intervention mapping. In step 2, EBIs targeting each indicator and determinant are evaluated for their acceptability to the ethno-cultural community, and only those perceived as acceptable are reviewed in step 3 to determine the modifications needed to 
Step 1: Assessing the ethno-cultural community's beliefs

1) Examine the target ethno-cultural community's understanding of the health problem

Have participants complete questionnaire to assess the relevance and importance of the indicators and determinants of the health problem (identified from literature) to the target ethno-cultural community

- Engage participants in a discussion to gain an understanding of the target ethno-cultural community's cultural beliefs and unique experiences of the health problem

2) Identify the indicators and determinants of the health problem that are of relevance to the target ethno-cultural community and that require intervention

Step 2: Examining interventions' fit with the community's beliefs

1) Select evidence-based interventions that address the indicators and determinants of the health problem of relevance to the target ethno-cultural community

2) Have participants rate the acceptability of the interventions

3) Engage participants in a discussion to clearly identify the interventions considered acceptable or desirable to the target ethno-cultural community, and to map these interventions relative to the respective indicator or determinant of the health problem

4) Identify interventions viewed favorably

Step 3: Adapting interventions

1) Review the information about each intervention viewed favorably (i.e. resources needed to implement the intervention, components, activities, mode and dose of delivery)

2) Engage participants in a semi-structured discussion to identify elements or aspects of the intervention that should be modified and how, in order to enhance its relevance and fit with the ethno-cultural community's beliefs

Figure 1. Steps of the Integrated Strategy for Cultural Adaptation of Interventions.

enhance their desirability and relevance to the community.

The three steps are undertaken in collaboration with representatives of the target ethno-cultural community experiencing the health problem. Representatives are selected if they: report that they currently have the health problem or they have successfully managed it; self-identify as belonging to the target ethno-cultural community; and are cognitively able to engage in a discussion in either their native and/or English language. Using an individual's native language is preferred because it facilitates the expression of beliefs and values in familiar and culturally relevant terminology.

The steps are implemented in one or more face-to-face, individual or group sessions. Ample time (estimated at 1 - 4 hours, based on our experience implementing the integrated strategy) should be allocated to: explain the activities in which the community representatives are to be engaged; collect quantitative and qualitative data while answering questions to avoid rushed responses; and offer a rest period to minimize the potential for response burden. Group sessions (3 - 4 hours) are expected to last longer than individual (1 - 2 hours) sessions because of the dialogue that takes place among the members to explore collective knowledge.

Group sessions have the advantage of providing opportunities for participants to exchange ideas; respond to each others' comments; question, clarify, and elaborate on ideas; and reach agreement that captures collective knowledge within a short time period [20]. The number and format of sessions to be held with community representatives is based on their preferences and ability to attend and stay actively engaged in a session of 3 - 4 hour duration; their comfort in a group discussion; and the logistics of finding a place and time convenient to all participants. If group sessions are used, it is advisable to run them with more than one group of participants representing the same community in order to enhance the transferability of the findings to the target ethno-cultural community. If individual sessions are used, it is advisable to have a total of $20-25$ par- 
ticipants. This sample size ensures that the collection and analysis of qualitative data are manageable while achieving data saturation, and facilitates the integration of quantitative results with the themes emerging from the qualitative data analysis [21].

The sessions are facilitated by persons with expert knowledge in the health problem and corresponding interventions, awareness of the target culture, and ability to converse in the native language; bicultural and bilingual persons are ideal to build rapport and promote disclosure [22]. The protocol for implementing the steps of the integrated strategy is detailed next.

\subsection{Step 1: Assessing the Ethno-Cultural Community's Beliefs}

This step is foundational to the cultural adaptation process as it aims to delineate the community's unique understandings and experiences of the health problem. In this step, the focus is on depicting the community's view of the health problem, which is influenced by its particular cultural beliefs. Accordingly, an understanding of the health problem should be generated before inquiring about the ethno-cultural community's beliefs.

Understanding of the health problem is generated from a review and synthesis of relevant sources. The understanding depicts the nature of the problem (what it is), its indicators (how it is manifested), and its determinants (what factors contribute to the problem) [14] [23]. Information on the problem is derived from pertinent conceptual, empirical and/or clinical sources (Table 1). The synthesis aims to integrate the findings obtained from these sources; it consists of comparing and contrasting the health problem's indicators and determinants proposed by theories or conceptual frameworks and supported by results of quantitative and qualitative research. The comparison is done to delineate indicators and determinants that reflect the same concept but may have been worded differently across sources. Consistent with the concept mapping process, a comprehensive list of indicators and determinants is generated and incorporated into a questionnaire.

The questionnaire identifies and describes each indicator and determinant of the health problem in simple and easy to understand lay language. The questionnaire is administered during the session to assess the relevance (i.e. extent to which the indicator or determinant is experienced by members of the community) and importance (i.e. extent to which the indicator or determinant significantly impacts members of the community) of the indicators and determinants to the target ethno-cultural community. The assessment is done on a 10-point numeric rating scale anchored by not at all (0) and very much (10) or five-point scale $(0=$ not relevant/important, $1=$ a little relevant/important, $2=$ somewhat relevant/important, $3=$ much relevant/important, $4=$ very much relevant/important). The rating scale is selected in consultation with representatives of the target ethno-cultural community. The questionnaire can be translated into the ethno-cultural community's native language following standard translation procedures to enhance comprehension of its content and improve the accuracy of 
Table 1. Sources of information on indicators and determinants of the health problem.

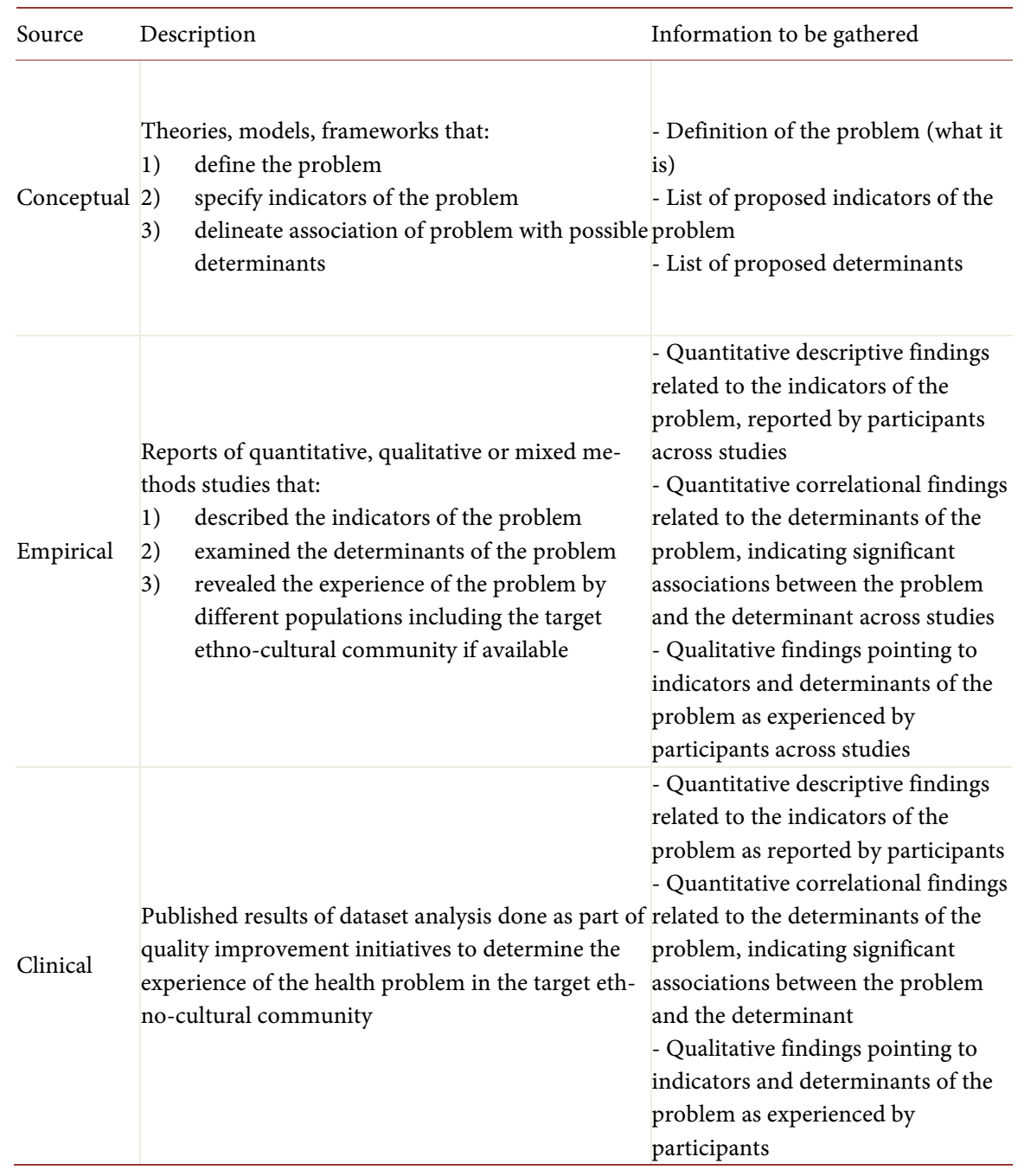

responses. In addition, open-ended questions are prepared to engage community representatives in a discussion aimed at clarifying the community's and/or its subgroups' beliefs about the health problem.

The individual or group session proceeds as follows:

1) Explain that the purpose of the session is to generate an understanding of the target ethno-cultural community's views and experiences of the health problem of interest.

2) Give an overview of the health problem by describing what it is (e.g. limited engagement in physical activity), its indicators (e.g. walking for less than $150 \mathrm{mi}-$ nutes per week) and determinants (e.g. lack of time, unsafe neighborhood), while emphasizing that this information has been obtained from pertinent research.

3) Distribute the questionnaire and provide specific instructions on how to rate the indicators and determinants relative to their relevance and importance to themselves and others in the target community who have the health problem (e.g. people, like you, who are Chinese and do not exercise). Provide a copy of the questionnaire (i.e. English or translated version) preferred by participants 
and assist them, as needed, in reading the descriptions of indicators and determinants, clarifying content, and understanding the rating scale.

4) Invite participants to individually complete the questionnaire. Clarify any point as needed.

5) Engage participants in a discussion to gain an understanding of the target ethno-cultural community's cultural beliefs and unique experiences of the health problem. Questions to guide the discussion include:

- Which indicators (i.e. things that tell you that the problem exists) do you and people in your community experience with the health problem? By community, we mean your cultural or ethnic group. Are there other indicators of the health problem in your community?

- Which factors do you believe most significantly contribute to the health problem as experienced by you and people of your community? How do these significant factors contribute to the problem? Are there other factors? How do these factors relate to the problem?

- Given your experience with the health problem, which indicators or factors are important to address to help resolve it? Which should be given priority? What makes them important? Are these indicators and factors important for all people in your community or are some more or less important for particular people of your community (e.g. men/women, young/older people)?

The quantitative (i.e. ratings) and qualitative (i.e. responses to open-ended questions) data are subjected to parallel mixed analysis, as proposed by Spillane et al. [24]. The quantitative ratings are analyzed descriptively to determine the most relevant and important (evidenced by high means and low variances) indicators and determinants. The transcripts of qualitative comments are content analyzed. The analyses are done within and across individual or group sessions. A matrix is used to facilitate the integration of quantitative and qualitative findings. The matrix represents the mean rating and corresponding codes for each indicator and determinant. Findings that converge across methods are integrated to identify the indicators and determinants (e.g. those having high mean ratings and codes reflecting their importance) that represent the target ethno-cultural community's understanding and experiences of the health problem. The quantitative and qualitative findings inform the selection of interventions that have been found effective in addressing the relevant indicators and determinants, and the examination of their congruence with the community's beliefs about the health problem and its treatment, as explained next.

\subsection{Step 2: Examining Interventions' Fit with the Community's Beliefs}

The goal of this step is to identify interventions that are either aligned or nonaligned with the target community's cultural beliefs related to the health problem. Aligned interventions are considered acceptable and viable in addressing the health problem and its indicators or determinants, as experienced by the community [1]. Non-aligned interventions may be disregarded, as they will not 
be sought, initiated and adhered to. Interventions that have some non-aligned aspects, such as their mode of delivery (e.g. in a group format) and some aligned aspects, such as their content (e.g. aerobic exercise) are subjected to further adaptation in step 3.

Preparation for this step involves selecting EBIs, understanding the theoretical underpinning of the EBIs, and developing a description for each EBI, the measure assessing its acceptability, and a set of open-ended questions to guide the discussion. Results of meta-analyses or systematic reviews inform the selection of EBIs found effective in managing the health problem, its indicators or determinants. EBIs are chosen regardless of the community in which their effectiveness was demonstrated. Effectiveness is evidenced by: 1) remission rate, that is, the percentage of patients reporting resolution of the problem, indicator or determinant targeted by the intervention; 2) statistically significant differences, between and within groups, in the experience of the problem, indicator or determinant (e.g. reduced level of severity); and/or 3) clinically meaningful improvement in the experience of the problem, indicator or determinant (e.g. medium or large effect size). In addition, a good understanding of the theoretical underpinning (i.e. specific elements, mechanism responsible for producing their effects, components and activities operationalizing the specific elements) and the protocol (i.e. strategies for carrying out the components and activities) guiding the implementation of the identified EBIs is sought by reviewing pertinent literature and/or discussing them with the intervention designers.

A description of each EBI is developed from a synthesis of information on the conceptualization, operationalization and effectiveness of the intervention, obtained from pertinent conceptual, empirical and clinical, published and grey sources. Conceptualization of, or theory underlying, the intervention clarifies the health problem or its indicator or determinant that the intervention addresses, the goal of the intervention, its specific elements, its non-specific elements, the short-term outcomes that mediate its effects on the ultimate outcomes that represent resolution or improvement in the experience of the health problem, as well as general well-being [19] [25].

Operationalization of an intervention relates to practical strategies for implementing it. Operationalization specifies: 1) the components and activities reflecting the specific elements that are to be implemented by the therapist and patients when applying the intervention; 2) the activities that reflect the non-specific elements (e.g. completing a diary, self-reflection on barriers to exercise) and mode or format for delivering the intervention; and 3) the dose of the intervention. Effectiveness reflects the benefits of the intervention. In addition, the potential risks (i.e. adverse reactions or discomforts) are identified.

The description presents this information about an EBI in simple language, easy to follow format, and as factual statements, as recommended by Tarrier, Liversidge and Gregg [26]. The description includes the name of the intervention (e.g. behavioral therapy); its goal or what it is set to achieve (e.g. promote physical activity); main components, activities and treatment recommendations 
to be followed (e.g. engage in physical activity 30 minutes per day, 5 days per week); mode and dose of delivery (e.g. four classes of 45 minutes each, given by the therapist once a week over a four-week period); benefits (e.g. found to improve physical function); and risks (e.g. minimal risks including tiredness and muscle pain).

The measure of acceptability is modified from the Treatment Perception and Preferences (TPP) scale [27]. The TPP contains a description of each EBI under consideration, followed by items to rate its appropriateness (i.e. extent to which it is reasonable in addressing the health problem, and suitable to one's lifestyle), benefits (i.e. extent to which it is effective in addressing the health problem in the short and long term), risks (i.e. extent to which adverse reactions or discomforts associated with the intervention are burdensome), and convenience (i.e. extent to which it is easy to carry out and adhere to). A five point scale, ranging from not at all (0) and very much (4) is used for the rating.

Examining the fit of the selected EBIs with the target ethno-cultural community's beliefs is done by assessing the EBIs' perceived acceptability to the community representatives [11]. This involves the following actions:

1) Explain that the purpose of the session is to get community's views of interventions that have been found effective in addressing the health problem, its indicators and/or determinants. Clarify that the interest is in determining whether or not the interventions are acceptable, that is, considered relevant, appropriate or potentially useful in managing the health problem as experienced by the community.

2) Review briefly what the health problem is, and its indicators and determinants that the community representatives considered relevant and important (based on step 1 results).

3) Clarify that research has shown that a number of interventions are successful in managing the health problem, its indicators and determinants. Present descriptions of these interventions in the TPP questionnaire.

4) Distribute the questionnaire and ask participants to follow along while the facilitator reads the description of one intervention. Address any questions participants may have so that they have a good grasp of what the intervention consists of.

5) Ask participants to individually rate the acceptability of the intervention by responding to the respective items on the TPP questionnaire. Remind participants to rate the intervention for its appropriateness, benefits, risks, and convenience to the target ethno-cultural community.

6) Once all participants have completed rating the intervention, engage them in a semi-structured discussion to further explore their perspectives on this intervention. The open-ended questions to use, adapted from Ayala and Elder [22] Bernal and Saez-Santiago [28] and Castro et al. [6] are:

- Overall, what do you think of this intervention? Is it acceptable to, or desired by people of your community to help them manage the health problem as they experience it? Is it acceptable to all people in your community or is it more or 
less acceptable for particular groups (e.g. men/women, young/older people)?

- What makes this intervention acceptable/unacceptable or desirable/undesirable? What specific aspects (e.g. what it consists of, how it is given) of the intervention are acceptable/unacceptable or desirable/undesirable?

- How does the intervention fit/not fit, with your community's views of the health problem and beliefs about its treatment? Would people of your community have the resources needed to carry out the intervention?

7) Repeat actions 4 to 6 for each of the remaining interventions.

8) Engage participants in a discussion to clearly identify the interventions considered acceptable or desirable to the target ethno-cultural community, and to map these interventions relative to the respective indicator or determinant of the health problem, as applicable. The open-ended questions guiding the discussion include:

- Of all the interventions you rated, which are, the most acceptable or desirable to people of your community? Which are not?

- Of the interventions you considered acceptable, which are the most appropriate or useful to manage the respective indicator or determinant.

The quantitative (i.e. ratings of interventions' acceptability) and qualitative (i.e. responses to open-ended questions) data analyses are comparable to those described in step 2. Convergent findings would indicate the EBIs viewed favorably or unfavorably. Favorable interventions are those with a mean rating $\geq 2$, which is the midpoint of the TPP rating scale, and those identified as congruent with the community's view of the health problem and beliefs about its treatment. These interventions are advanced to the next step for possible adaptation.

\subsection{Step 3: Adapting Interventions}

The goals of this step are to specify aspects of each EBI rated as acceptable and to delineate the changes to be made. To reiterate, interventions that are rated in step 2 as unacceptable and require omission or significant modification of components and activities that reflect their specific elements, are inappropriate or unviable options for managing the health problem in the target ethno-cultural community. Interventions viewed favorably are discussed in step 3. However, no changes are made to the interventions' components and activities that operationalize its specific elements because doing so may alter the elements comprising the intervention and weaken its effectiveness in improving the outcomes [3] [25] [29].

Accordingly, comprehensive knowledge of the interventions' conceptualization is critical in guiding their adaptation. Table 2 presents strategies for gaining this knowledge; the information obtained from different sources are compared and contrasted to identify commonly and consistently reported specific elements of the intervention. Adaptation can be done to: the presentation of content (e.g. link topics to cultural beliefs, use culturally appropriate metaphors); the strategy for implementing treatment recommendations (e.g. substitute jogging with cultural dance as a means for engaging in physical activity); non-specific activities (e.g. formation of a women's walking group); mode of delivery (e.g. having a personal trainer of the same sex); and dose of the intervention (e.g. performing 
Table 2. Strategies to gain knowledge of intervention conceptualization (adapted from Sidani and Braden, 2011).

\begin{tabular}{|c|c|c|}
\hline Strategy & Source & Information to be gathered \\
\hline $\begin{array}{l}\text { Review } \\
\text { theoretical/ } \\
\text { conceptual } \\
\text { literature }\end{array}$ & $\begin{array}{l}\text { Publications } \\
\text { (journal, book) } \\
\text { presenting: }\end{array}$ & \\
\hline \multirow{4}{*}{$\begin{array}{l}\text { Review } \\
\text { empirical } \\
\text { literature }\end{array}$} & $\begin{array}{l}\text {-Theory/conceptual } \\
\text { framework underlying } \\
\text { the intervention }\end{array}$ & $\begin{array}{l}\text {-Theory/conceptual framework } \\
\text { may suggest strategies to address the health } \\
\text { problem, its indicators and determinants. } \\
\text { The strategies may represent specific } \\
\text { elements of the interventions. } \\
\text {-Theory/conceptual framework identifies the } \\
\text { elements that characterize the intervention } \\
\text { and distinguish it from other interventions } \\
\text { (i.e. unique features that define the } \\
\text { intervention) and that are responsible for its } \\
\text { effects in addressing the health problem }\end{array}$ \\
\hline & $\begin{array}{l}\text { Publication (journals, books, } \\
\text { conference proceedings, } \\
\text { online slide presentations) } \\
\text { presenting findings of quantitative, } \\
\text { qualitative or mixed method } \\
\text { studies that evaluate } \\
\text { the intervention }\end{array}$ & \\
\hline & $\begin{array}{l}\text {-Methodological part of the study } \\
\text { report }\end{array}$ & $\begin{array}{l}\text {-Theory/conceptual framework guiding the } \\
\text { intervention and/or the study identifies } \\
\text { specific elements of intervention } \\
\text {-Description of the intervention delineates } \\
\text { the core components that operationalize the } \\
\text { specific elements, the activities performed, } \\
\text { and the mode of delivery. Some activities and } \\
\text { mode of delivery represent non-specific } \\
\text { elements of the intervention }\end{array}$ \\
\hline & -Empirical part of the study report & $\begin{array}{l}\text {-Results and discussion sections delineate } \\
\text { aspects of the intervention and/or its } \\
\text { implementation that were carried out with } \\
\text { fidelity and contributed to the outcomes }\end{array}$ \\
\hline $\begin{array}{l}\text { Review } \\
\text { intervention pro- } \\
\text { tocol/manual }\end{array}$ & $\begin{array}{l}\text { Intervention protocol may be } \\
\text { published in clinically-oriented } \\
\text { journals or books. } \\
\text { Intervention manual may } \\
\text { have to be requested/purchased } \\
\text { from the interventionists }\end{array}$ & $\begin{array}{l}\text { These sources usually provide an overview of } \\
\text { the theory/conceptual framework underlying } \\
\text { the intervention, explain the specific } \\
\text { elements, and specify the activities that } \\
\text { reflect the specific elements and } \\
\text { the non-specific elements }\end{array}$ \\
\hline $\begin{array}{l}\text { Discuss with the } \\
\text { interventionists }\end{array}$ & $\begin{array}{l}\text { Interventionists include persons who: } \\
\text {-Originally developed the } \\
\text { intervention } \\
\text {-Evaluated the intervention } \\
\text { effects in one or more studies } \\
\text {-Use the intervention } \\
\text { in the practice setting }\end{array}$ & $\begin{array}{l}\text { Discussion, done formally (e.g. in mixed } \\
\text { method study to examine the } \\
\text { implementation and effects of the } \\
\text { intervention) or informally, focuses on } \\
\text { eliciting interventionists' perspective on what } \\
\text { constitute the specific elements responsible } \\
\text { for the intervention's effects, and the } \\
\text { non-specific elements of the intervention }\end{array}$ \\
\hline
\end{tabular}


the physical activity in two 15 minute sessions, rather than one 30 -minute session, per day).

Adaptation demands adequate preparation. In addition to gaining in-depth understanding of the conceptualization and operationalization of the interventions, a logic model is developed for each intervention. A logic model is a visual illustration (table or diagram) of the intervention's operations and effects. It presents information on the resources needed to implement the intervention, the process of delivering the intervention, and the short and long term outcomes [15] [23] as illustrated in Table 3. For each intervention, the logic model clarifies: 1) the human and material resources that people need to carry out the intervention in daily life; 2) the key components and activities, mode and dose of delivery, which are represented with an account of the content to be covered, the activities to be performed, and the treatment recommendations to be discussed in each intervention session; and 3) the short and long term outcomes that are empirically supported (for details on the development of logic models, refer to [10] [30]). The risks associated with the intervention are also listed. A slide presentation of the intervention's logic model is prepared.

Table 3. Example of logic model for a multi-component intervention targeting physical activity.

\begin{tabular}{|c|c|c|c|c|c|}
\hline $\begin{array}{l}\text { Health } \\
\text { problem }\end{array}$ & Resources & Processes & $\begin{array}{c}\text { Short-term } \\
\text { outcome }\end{array}$ & $\begin{array}{c}\text { Long-term } \\
\text { outcome }\end{array}$ & Risk \\
\hline $\begin{array}{l}\text { Low } \\
\text { engagement } \\
\text { in physical } \\
\text { activity }\end{array}$ & $\begin{array}{l}\text { 1.Human: } \\
\text {-Interventionists (e.g. } \\
\text { Physical } \\
\text { therapist or nurse) } \\
\text { 2.Material: } \\
\text {-Room } \\
\text { (to hold sessions) } \\
\text {-Booklet (presenting } \\
\text { information covered } \\
\text { in component 1) } \\
\text {-Pedometer } \\
\text { (to monitor physical } \\
\text { activity) } \\
\text { 2) } \\
\text {-Paper and pen } \\
\text { (to write down } \\
\text { action plan in } \\
\text { component }\end{array}$ & $\begin{array}{l}\text { Behavioral Therapy to } \\
\text { promote physical } \\
\text { activity } \\
\text { Component } 1 \text { : } \\
\text { Education } \\
\text {-Factors that contribute to } \\
\text { physical inactivity } \\
\text {-Consequences of } \\
\text { physical inactivity } \\
\text {-Importance of being } \\
\text { physically active } \\
\text { Component } 2 \text { : Implemen- } \\
\text { tation of recommended } \\
\text { physical activity } \\
\text {-Engagement in } \\
\text { physical activity, } 30 \text { mi- } \\
\text { nutes per day, } 5 \text { days per } \\
\text { week } \\
\text {-Types of physical } \\
\text { activity to perform } \\
\text {-Action plan for carrying } \\
\text { out the recommended } \\
\text { physical activity } \\
\text {-Monitoring physical } \\
\text { activity performance } \\
\text {-Discussion of barriers and } \\
\text { facilitators, and strategies to } \\
\text { overcome barriers }\end{array}$ & $\begin{array}{l}\text { Increased } \\
\text { knowledge } \\
\text { about } \\
\text { importance of } \\
\text { physical } \\
\text { activity } \\
\text { Engagement } \\
\text { in physical } \\
\text { activity at } \\
\text { recommended } \\
\text { level }\end{array}$ & $\begin{array}{l}\text { Improved } \\
\text { physical } \\
\text { function }\end{array}$ & $\begin{array}{l}\text { Tiredness } \\
\text { Muscle } \\
\text { pain }\end{array}$ \\
\hline
\end{tabular}


Adaptation of the intervention follows these actions:

1) Explain that the purpose of the session is to discuss aspects of the evidence-based interventions, rated favorably by representatives of the target ethno-cultural community (i.e. acceptable and congruent with the cultural beliefs) and those that need to be changed to enhance their congruence.

2) Review briefly what the health problem is, its indicators and determinants that the community representatives considered relevant and important, and the respective interventions they viewed favorably.

3) Begin with one intervention. State its name and present the slide illustrating its logic model.

4) Review the information presented in the logic model while emphasizing the resources and the process (i.e. components, activities, mode and dose of delivery) to implement the intervention. Clarify any aspect of the intervention, as needed so that participants understand the intervention.

5) Explain that some aspects of the intervention cannot be changed because they are essential for the intervention to be effective in addressing the health problem. List the intervention components and activities that operationalize the essential elements. Clarify that: 1) completely eliminating these components and activities means that this intervention may not be a viable and effective option for the community; 2) very minor changes are allowed in the content or topics covered in these components and activities to enhance their relevance to the community's beliefs (e.g. omitting discussion of a barrier that is not encountered by members of the community and substituting it with another viewed as significant in preventing engagement in physical activity); 3) changes can be made in the way the components and activities are implemented (e.g. composition of the group formed to provide support when performing physical activity; type of physical activity to perform).

6) Engage participants in a semi-structured discussion to answer the openended questions adapted from Ayala et al. [22], Bernal and Saez-Santiago [28], Cabassa et al. [15] and Castro et al. [6].

- Overall, does this intervention fit with, or is congruent with your community's view of the health problem and beliefs about its treatment?

- What aspects of the intervention do/do not quite fit with your cultural beliefs?

a) Consider the content, topics, mode and dose of delivery, daily activities, and treatment recommendations. Which of these are acceptable/unacceptable? Which do/do not fit with cultural beliefs of your community? Which do/do not fit with people's abilities to carry them out? Which are convenient/inconvenient (i.e. easy to apply and adhere to)? Are there some groups (e.g. men/women, young/older people) from your community who may find these topics and activities more or less acceptable, desirable or convenient?

b) Consider the mode and dose of delivery. Is the format (e.g. individual or group session) in which the intervention is given acceptable/unacceptable, appropriate/inappropriate, convenient/inconvenient, more or less useful in helping 
people get the information/knowledge and ability/skills needed to successfully manage the health problem or its indicators/determinants? Are the number and length of the sessions appropriate/inappropriate, convenient/inconvenient, enough/not enough to help people get the information/knowledge and ability/ skills needed to successfully manage the health problem or its indicators/determinants? Would people be able to attend all the sessions as planned? Is the setting in which the intervention is to be given an issue?

c) Consider the way in which the content and activities are presented. Is language an issue? Are there ideas/words that should be avoided/modified/replaced by particular notions or terms that are more culturally appropriate? Are there resources that people would need to carry out the treatment recommendations in their daily life? Would people have access to these materials?

- What factors may hinder/make it difficult for people to apply the intervention/treatment recommendations in daily life?

- What would facilitate/make it easier for people of your community to apply the intervention/treatment recommendations in daily life?

- What aspects of the intervention should be changed to make the intervention acceptable to people of your community? What specific changes need to be made to the intervention's components, content or topics, activities, treatment recommendations, language, context of delivery, mode, dose, resources?

7. Repeat actions 3 to 6 for each intervention under consideration.

The transcripts of the discussion are content analyzed to determine the modifications that should be made to enhance the fit of each intervention with the community's cultural beliefs. Suggestions for adaptation consistently made across groups of participants are identified and integrated into the operationalization (i.e. logic model, protocol, and manual) of each intervention.

\section{Discussion}

The necessity and importance of culturally adapting EBIs are well recognized [6]. The aim is to generate a version of these interventions that fits with the cultural beliefs, values or norms of the target ethno-cultural community while maintaining the interventions' specific elements that are responsible for their effectiveness [3] [31]. As such, the adaptation process should be informed by a lucid understanding of the conceptualization and operationalization of the interventions. This knowledge is critical in identifying the components and activities that represent the interventions' specific elements, and in guiding possible modifications. Cumulating evidence indicates that culturally adapted interventions are viewed favorably by community members, and have been shown to enhance people's initiation, engagement and adherence to intervention recommendations, and to improve outcomes [3]. The availability of culturally adapted interventions has the potential to increase an ethno-cultural community's access to health care and reduce health disparities [32].

To successfully align interventions with a community's cultural beliefs, adaptation should be done carefully to preserve the specific elements of the interven- 
tions that are responsible for improving outcomes, while accounting for the community's cultural beliefs [9]. The integrated strategy provides a detailed protocol that specifies what is to be accomplished and how. The strategy integrates concept and intervention mapping, and uses mixed methods to gather data from representatives of the target ethno-cultural community. Concept and intervention mapping represent effective and efficient approaches to systematically and actively engage community representatives in clarifying their cultural beliefs about the health problem and their acceptability of EBIs [15]. The use of mixed methods provides opportunities for community representatives to: have a say in reflecting on their views about the problem and the interventions (through rating the importance of the problem determinants and indicators, and the acceptability of the interventions); quantify and expand on their cultural beliefs, values and norms; and reach a collective agreement on aspects of the interventions that need to be adapted and how to adapt them. Overall, the proposed protocol is a useful reference for planning and carrying out the cultural adaptation process in a consistent manner; inconsistency in its application may yield differences in the conceptualization and operationalization of interventions adapted for the same ethno-cultural community. These differences may threaten the effectiveness of adapted interventions and restrict the replication of beneficial outcomes within the same community.

Implementing the protocol may be demanding and time consuming. It requires collaboration among researchers with experience in intervention design and adaptation [15] as well as adequate preparation. Preparation includes: the synthesis of evidence on the health problem and respective EBIs; generation of questionnaires to assess the importance of determinants and indicators of the problem and acceptability of the interventions; formulation of the open-ended questions and prompts; recruitment of community representatives; and availability of bilingual and/or bicultural session facilitators. The sessions, whether done individually or in a group, are long and cognitively taxing for the facilitators and the community representatives. The facilitators have to follow the protocol while clarifying its steps and simplifying, as needed, the information to ensure adequate understanding of the health problem and the interventions. The facilitators need to actively engage all representatives in the planned activities while managing group dynamics and asking for expansion on specific ideas to reach group agreement. Community representatives are requested to participate in multiple activities (rating and discussing determinants and indicators of the problem; rating and discussing interventions; identifying aspects of the interventions to be adapted and suggesting ways to modify them). The representatives need to recognize and bring forth their cultural beliefs (which may often be tacit knowledge), and they need to develop an understanding of the interventions with which they may not be familiar. Furthermore, attending a long session may not be convenient. Therefore, two sessions can be organized with each group of community representatives. The first session focuses on exploring the cultural beliefs about the health problem, whereas the second session involves assessing 
the interventions' acceptability and modifying them to align the interventions with cultural beliefs. Despite these limitations, the protocol has the potential to facilitate rigorous adaptation of EBIs and enhance their fit with the cultural beliefs of diverse communities.

Adapted interventions are moved to the next phase of the cultural adaptation process. They are evaluated for their feasibility, acceptability and ability to induce the hypothesized changes in outcomes, prior to determining their effectiveness in addressing the health problem as experienced by the target ethno-cultural community.

\section{Conclusion}

Cultural adaptation of EBIs follows a systematic process to align interventions with the beliefs of the target ethno-cultural community. The proposed integrated strategy extends the systematic process by detailing the protocol for carrying it out. Its strength relies on the use of concept and intervention mapping, and mixed methods for exploring the community's cultural beliefs, acceptability and modifications of the interventions. Additional research is needed to determine the utility of the integrated strategy in culturally adapting health interventions.

\section{References}

[1] Bernal, G., Jimenez-Chafey, M. and Domenech Rodriguez, M.M. (2009) Cultural Adaptation of Treatments: A Resource for Considering Culture in Evidence-Based Practice. Professional Psychology. Research and Practice, 40, 361-368. https://doi.org/10.1037/a0016401

[2] Kreuter, M.W. and Haughton, L.T. (2006) Integrating Culture into Health Information for African American Women. American Behavioral Science, 49, 794-811. https://doi.org/10.1177/0002764205283801

[3] Barrera Jr., M., Castro, F.G., Strycker, L.A. and Toobert, D.J. (2014) Cultural Adaptations of Behavioral Health Interventions: A Progress Report. Journal of Consult ing and Clinical Psychology, 81, 196-205. https://doi.org/10.1037/a0027085

[4] Givens, J.L, Houston, T.K., van Voorhees, B.W., Ford, D.E. and Cooper, L.A. (2007) Ethnicity and Preferences for Depression Treatment. General Hospital Psychiatry, 29, 182-191. https://doi.org/10.1016/j.genhosppsych.2006.11.002

[5] Cardemill, E.V. (2010) Cultural Adaptations to Empirically Supported Treatments: A Research Agenda. Scientific Review of Mental Health Practice, 7, 8-21.

[6] Castro, F.G., Barrera Jr., M. and Steiker, L.K.H. (2010) Issues and Challenges in the Design of Culturally Adapted Evidence-Based Interventions. Annual Review of Clinical Psychology, 6, 213-239. https://doi.org/10.1146/annurev-clinpsy-033109-132032

[7] Marsiglia, F.F. and Booth, J.M. (2014) Cultural Adaptation of Interventions in Real Practice Settings. Research on Social Work Practice, 25, 423-432. https://doi.org/10.1177/1049731514535989

[8] Barrera, M. and Castro, F.G. (2006) A Heuristic Framework for the Cultural Adaptation of Interventions. Clinical Psychology. Science and Practice, 13, 311-316. https://doi.org/10.1111/j.1468-2850.2006.00043.x

[9] Castro, F.G., Barrera Jr., M. and Martinez Jr., C.R. (2004) The Cultural Adaptation of Prevention Interventions: Resolving Tensions between Fidelity and Fit. Preven- 
tion Science, 5, 41-45. https://doi.org/10.1023/B:PREV.0000013980.12412.cd

[10] Center for Disease Control Website. http://www.cdc.gov/eval/resources.htm\#logic\%20model

[11] Ferrer-Wreder, L., Sundell, K. and Mansoory, S. (2012) Tinkering with Perfection: Theory Development in the Intervention Cultural Adaptation Field. Child \& Youth Care Forum, 41, 149-171. https://doi.org/10.1007/s10566-011-9162-6

[12] Papas, R.K., Sidle, J.E., Martino, S., Baliddawa, J.B., Songole, R., Omolo, O.E. and Maisto, S.A. (2010) Systematic Cultural Adaptation of Cognitive Behavioral Therapy to Reduce Alcohol Use among HIV-Infected Outpatients in Western Kenya. AIDS and Behavior, 14, 669-678. https://doi.org/10.1007/s10461-009-9647-6

[13] Wingwood, G.M. and DiClemente, R.J. (2008) The ADPAT-ITT Model. A Novel Method of Adapting Evidence-Based HIV Interventions. Journal of Acquired Immune Deficiency Syndrome, 47, S40-S46.

[14] Sidani, S. and Braden, C.J. (2011) Design, Evaluation, and Translation of Nursing Interventions. Wiley-Blackwell, Ames, IA. https://doi.org/10.1002/9781118785553

[15] Cabassa, L.J., Druss, B., Wang, Y. and Lewis-Fernández, R. (2011) Collaborative Planning Approach to Inform the Implementation of a Healthcare Manager Intervention for Hispanics with Serious Mental Illness: A Study Protocol. Implementation Science, 6, 80. https://doi.org/10.1186/1748-5908-6-80

[16] Sidani, S., Epstein, D., Bootzin, R., Moritz, P. and Miranda, J. (2009) Assessment of Preferences for Treatment: Validation of a Measure. Research in Nursing \& Health, 32, 419-431. https://doi.org/10.1002/nur.20329

[17] Burke, J.G., O’Campo, P., Peak, G.L., Gielen, A.C., McDonnell, K.A. and Trochim, W.M. (2005) An Introduction to Concept Mapping as a Participatory Public Health Research Method. Qualitative Health Research, 15, 1392-1410. https://doi.org/10.1177/1049732305278876

[18] Kok, G., Schaalma, H., Ruiter, R.A.C., van Empelen, P. and Brug, J. (2004) Intervention Mapping: A Protocol for Applying Health Psychology Theory to Prevention Programmes. Journal of Health Psychology, 9, 85-98. https://doi.org/10.1177/1359105304038379

[19] Sidani, S. (2015) Health Intervention Research: Advances in Research Design and Methods. Sage, London.

[20] Morgan, D.L. and Krueger, R.A. (1993) When to Use Focus Groups and Why. In: Morgan, D.L., Eds., Successful Focus Groups. Advancing the State of the Art, Sage Publications, New York, 3-19. https://doi.org/10.4135/9781483349008.n1

[21] Creswell, J.W. and Plano Clark, V.L. (2011) Designing and Conducting Mixed Methods Research. Sage Publications, Inc., Thousand Oaks, CA.

[22] Ayala, G.X. and Elder, J.P. (2011) Qualitative Methods to Ensure Acceptability of Behavioral and Social Interventions to the Target Population. Journal of Public Health Dentistry, 71, S69-S79. https://doi.org/10.1111/j.1752-7325.2011.00241.x

[23] Cabassa, L.J., Gomes, A.P., Meyreles, Q., Capitelli, L., Younge, R., Drafatsi, D., Alvarez, J., Manrique, Y. and Lewis-Fernández, R. (2014) Using the Collaborative Intervention Planning Framework to Adapt a Health-Care Manager Intervention to a New Population and Provider Group to Improve the Health of People with Serious Mental Illness. Implementation Science, 9, 178.

https://doi.org/10.1186/s13012-014-0178-9

[24] Spillane, J.P., Pareja, A.S., Dorner, L., Barnes, C., May, H., Huff, J. and Camburn, E. (2010) Mixing Methods in Randomized Controlled Trials (RCTs): Validation, Contextualization, Triangulation, and Control. Educational Assessment, Evaluation and 
Accountability, 22, 5-28. https://doi.org/10.1007/s11092-009-9089-8

[25] Kilbourne, A.M., Neumann, M.S., Pincus, H.A., Bauer, M.S. and Stall, R. (2007) Implementing Evidence-Based Interventions in Health Care: Application of the Replicating Effective Programs Framework. Implementation Science, 2, 42-51. https://doi.org/10.1186/1748-5908-2-42

[26] Tarrier, N., Liversidge, T. and Gregg, L. (2006) The Acceptability and Preference for the Psychological Treatment of PTSD. Behavior Research and Therapy, 44, $1643-$ 1656.

[27] Sidani, S., Epstein, D.R., Miranda, J. and Fox, M. (2016) Psychometric Properties of the Treatment Perception and Preferences Scale. Clinical Nursing Research, 25, 119.

[28] Bernal, G. and Saez-Santiago, E. (2006) Culturally Centered Psychosocial Interventions. Journal of Community Psychology, 34, 121-132. https://doi.org/10.1002/jcop.20096

[29] Aarons, G.A., Green, A.E., Palinkas, L.A., Self-Brown, S., Whitaker, D.J., Lutzker, J.R., Silvosky, J.F., Hecht, D.B. and Chaffin, M.J. (2012) Dynamic Adaptation Process to Implement an Evidence-Based Child Maltreatment Intervention. Implementation Science, 7, 32. https://doi.org/10.1186/1748-5908-7-32

[30] Taylor-Powell, E. and Henert, E. (2008) Developing a Logic Model: Teaching and Training Guide. University of Wisconsin, Extension Cooperative Extension.

[31] Thompson, V.L.S., Johnson-Jennings, M., Baumann, A.A. and Proctor, E. (2015) Use of Culturally Focused Theoretical Frameworks for Adapting Diabetes Prevention Programs: A Qualitative Review. Preventing Chronic Disease, 12, Article ID: 140421. https://doi.org/10.5888/pcd12.140421

[32] Mier, N., Ory, M.G. and Medina, A.A. (2010) Anatomy of Culturally Sensitive Interventions Promoting Nutrition and Exercise in Hispanics: A Critical Examination of Existing Literature. Health Promotion Practice, 11, 541-554. https://doi.org/10.1177/1524839908328991

Scientific Research Publishing

\section{Submit or recommend next manuscript to SCIRP and we will provide best} service for you:

Accepting pre-submission inquiries through Email, Facebook, LinkedIn, Twitter, etc. A wide selection of journals (inclusive of 9 subjects, more than 200 journals)

Providing 24-hour high-quality service

User-friendly online submission system

Fair and swift peer-review system

Efficient typesetting and proofreading procedure

Display of the result of downloads and visits, as well as the number of cited articles

Maximum dissemination of your research work

Submit your manuscript at: http://papersubmission.scirp.org/

Or contact health@scirp.org 